A. AURUM, C. WOHLIN AND A. PORTER, "ALIGNING SOFTWARE PROJ ECT DECISIONS: A CASE STUDY, INTERNATIONAL J OURNAL OF SOFTWARE ENGINEERING AND KNOWLEDGE ENGINEERING, VOL. 16, NO.6, PP. 795-818, 2006. 


\title{
ALIGNING SOFTWARE PROJECT DECISIONS: A CASE STUDY
}

\author{
AYBÜKE AURUM \\ School of Information Systems, Technology and Management, University of New South Wales, Sydney, 2052 NSW Australia, \\ aybuke@unsw.edu.au \\ CLAES WOHLIN \\ Department of Systems and Software Engineering, School of Engineering, Blekinge Institute of Technology \\ Box 520, SE-372 25 Ronneby, Sweden, \\ Claes.Wohlin@bth.se \\ ANDREW PORTER \\ School of Information Systems, Technology and Management, University of New South Wales, Sydney, 2052 NSW Australia,
}

\begin{abstract}
Recent research in software engineering has highlighted the need to ensure alignment between business objectives, customer requirements and product development. If the business is to meet its strategic objectives, the Requirements Engineering (RE) activities must be executed in a manner such that they support these higher level objectives. A clear alignment between RE activities and the strategic objectives of the organization should underscore the merit of IT investment and the opportunities for competitive advantage that can be pursued as a result by the organization. This research begins with a detailed investigation of the complexity of decision-making during RE activities on business, product and project levels. Secondly, it investigates 'in-project' level RE decisions and provides empirical findings from an industrial case study. The findings show that RE project related decisions are influenced by business and product requirements. Although, the software developers in this study recognized the importance of developing software products that meet customer requirements and that are aligned with business objectives, they found it difficult to achieve this when the customer was external and the communication was a major problem. A lack of understanding the business strategy and a lack of communication between business, product and project level stakeholders were among the problems that are highlighted by this study.
\end{abstract}

Keywords: Requirements engineering decisions, alignment of decisions, project decisions, business objectives, product decisions

\section{Introduction}

For many years, the study of decision-making was a minor field of endeavour for researchers studying software engineering (SE) and, in particular, requirements engineering (RE). Over the past decade however, there has been remarkable growth in research about decision-making from a wide variety of management and social science perspectives in SE. This article is about the decision-making process in RE activities.

Why is there interest in studying decision-making in RE? The RE process is a complex one and an understanding of how stakeholders select requirements is an ideal starting point for the development of methodologies which facilitate and improve the decision-making process and its outcomes [33, 34]. There are several challenges to overcome during the decision-making process for selecting requirements. Firstly, it is wellknown that not all requirements are equally important. Thus, there are considerations for a stakeholder to bear in mind when determining the relative importance of such requirements. Further, once a requirement is identified for inclusion in a software product, a stakeholder may be required to select one of the available alternatives for implementing that requirement. To facilitate the selection of requirements and their subsequent implementation, stakeholders need an appreciation of the economic implications of their decisions in the early stages of the development process. This is especially the case when developing new products in which product attributes are complex and difficult to characterize during the initial development process $[8,14]$. Furthermore, it is crucial to ensure that the requirements meet business goals and are aligned with business processes. SE literature has emphasized the importance of generating products that meet customer requirements and that are aligned with strategic business goals [27].

We believe that the impact of RE decisions can be observed in four levels, namely business, stakeholders, project, and product levels. Firstly, making good decisions will probably lead to a better business e.g. ability to sell the product. Secondly, a well defined decision-making process, and well informed stakeholders about the decision and the outcome of the decisions will satisfy stakeholders. Thirdly, good RE decisions are positively 
related to project cost effectiveness i.e. the ability of achieving a project within budget and on time. Finally, making good RE decisions will lead to a sense of satisfaction with the technical and value judgments about the product, and will lead to higher quality software products in general. Furthermore, it will also provide an opportunity for the product to continue evolving in the future.

RE decisions are inextricably intertwined with business, product and project decisions as software products have a major influence on system cost, schedule and value [6, 7]. These decisions are dependent upon the interaction between the individual stakeholders and the stakeholder group's expectations for how future businesses will evolve over time. These decisions may, for example, involve calculations of risk at business, project and product levels as well as consideration of return on investment. Some decisions are very complex given that they have multi-dimensional outcomes that cannot be compared objectively to one another. Hence, it is important to provide decision support to decision makers so that they can apply their preferences to a decision problem in a reasonable and reliable way. This is especially the case when dealing with unstructured or semistructured problems where multiple stakeholders are involved, and where there is uncertainty regarding the reliability or comprehensiveness of information drawn upon during decision-making [20]. The availability of reliable information sources is a key component of the decision-making process. The implication of this is that good decision support for RE activities should take the issues that we introduced above into consideration.

In order to meet business objectives and align with business processes, we argued [7] that RE decisions need to be considered a) in terms of product, project and business (organizational) decisions, and b) across three management levels i.e. strategic, tactical and operational levels. We examined the complex decision-making process which is inherent in RE activities on different managerial levels and presented a conceptual model that described and clarified the entities involved in the decision-making process in RE activities.

The objective of this paper is to provide a comprehensive analysis of the RE decision-making activities in a software project. In this paper we continue addressing the problem of how to improve the identification and support of requirements decisions. We investigate RE decisions in the context of software development projects using an industry-based case study, we provide empirical data and then present our findings. This research study is significant given that, to the best of our knowledge, there is no existing study that specifically focuses on the $\mathrm{RE}$ decision-making activities in a software development project. By contributing to the understanding of decision-making activities in RE through the use of an in-depth case study, it is envisaged that this will facilitate a better understanding of RE in general, and this should lead to more successful IT projects.

This paper proceeds by first describing and discussing briefly the concepts related to the decision-making process in RE. In Section 3, a RE decision framework, which emphasizes three different perspectives, is presented. In Section 4 a detailed description of the case study is provided. Section 5 describes the research objectives and the method of data collection. Section 6 presents the data analysis and discussion. A summary of findings, conclusion and future work is presented in Section 7.

\section{Background}

Decision-making is recognized as an activity that involves intelligence, design and choice phases [29]. Anthony [3] notes three types of decision-making activities in organizations based on the purpose of management activities: strategic planning, management control and operational control. In addition to the above, managerial decisions may have unstructured, semi-structured and structured decision components. Structured problems are more open to quantitative techniques, whereas many unstructured or semi-structured problems are solved by qualitative means using analytical tools. Decision makers apply intuition, experience, and their beliefs when solving such problems [15]. Furthermore, when dealing with structured problems, a decision maker always needs to employ his/her own intuitive assessment in addition to analytical tools.

\subsection{Decision-making in $R E$}

Each project begins with a statement of requirements. RE decisions include a selection of the requirements of the product to be developed, the choice of methods and tools for different activities, the identification of 
stakeholders, the feasibility, validation, and prioritization of requirements, and the selection of requirements for implementation during release planning. Key decisions during the management of $\mathrm{RE}$ activities include decisions on product scoping or activity releases, planned benefits of the product, deciding which type of quality assurance technique is likely to give the best return on investment etc. In many situations conflict is inherent in requirements, thus some requirements need to be negotiated between stakeholders. The discussion between stakeholders is generally informal and unstructured, involving many decisions and review points with iterations and design implementation activities. These decisions are generally continuous and, in numerous cases, uncertainties in the final cost, schedule, performance, and functionality are inevitable.

Evans et al., [13] emphasize the importance of recognizing requirements as design decisions in order to achieve a fully integrated software system. Aurum and Martin [4] point out the resemblance between the activities involved in organizational decision-making, and those in the requirements engineering process, by referring to classical decision-making theory. They present an approach that facilitates problem solving activities for requirements engineers. Regnell et al. [25] discuss descriptive and prescriptive research issues for understanding and supporting the requirements engineering decision-making process. Rolland et al. [26] introduce a decision-oriented process meta-model that aims to capture not only how activities are performed during the RE process but also why and when these activities are performed. In general, researchers agree that the RE process is a semi-structured or unstructured complex decision-making process [4, 13]. Aurum and Wohlin [5] describe the fundamental nature of RE activities in the decision-making process and examine the integration of classical decision-making models into the RE process. Alenljung and Persson [1] discuss RE decisions from a decision theory perspective. Jiang and Eberlein [16] present a decision support model for the selection of RE processes models and techniques. Ngo-The and Ruhe [22] have written a detailed analysis of current research related to RE decisions.

\subsection{The Role of Stakeholders and Information in RE Decisions}

$\mathrm{RE}$ decisions are made by stakeholders. In essence, RE aims at transforming potentially incomplete, inconsistent and conflicting stakeholder goals into a high quality complete set of requirements. Typical stakeholders are product managers, various types of users and administrators from the client side, and the software team members from the software development side. Note that this view is somewhat limiting when considering software development for markets. In other words, for market-driven software products customers may not necessarily have direct involvement in the development process. Furthermore, the degree of stakeholder involvement and the types of stakeholders may vary according to the software product and the project. For example, the communication style e.g. the negotiation process between the stakeholders, will be quite different for in-house software development than for customer-specific software development or the alignment of business objectives with customer requirements. The importance of stakeholder involvement in RE activities is widely accepted given that the quality of the software product is largely determined by the accurate identification of stakeholder needs [9].

In many situations, incoming information to a stakeholder is external, ambiguous and its accuracy is questionable. It is crucial to seek information about potentially important aspects of the problem. This leads to a way of relating pieces of information to each other in order to better understand the product in a business context. As a result, developers have an improved understanding of the company business, as well as business opportunities and they analyze, evaluate and select strategies to eliminate, manage or mitigate potential risks. We have written other discussion on information requirements for RE decisions [5, 7].

\subsection{Business Objectives, Business Processes and Business Rules}

An important issue in $\mathrm{RE}$ is that $\mathrm{RE}$ decisions need to meet business goals and must align with business objectives.

Business processes are structured organizational guidelines that illustrate how to meet business objectives. Business objectives or business goals show how an organization will direct its efforts. When developing 
software, it is important that the software product meets business needs [11]. Dawson [12] points out the relationship between the software process and business process, as most business processes involve the extensive use of software products that are embedded in business environments. On the one hand, Champion and Moores [10] point out that requirements which are elicited from stakeholders will inevitably have an influence on business rules and procedures. On the other hand, if business objectives or procedures are decided prior to requirements elicitation then software requirements will be influenced by these practices. Lubars et al., [19] discusses how important it is to understand the effect of business changes and to record decisions made about their impact on requirements. Neumann-Alkier [21] argues that software applications need to be well aligned with business strategies of organizations, especially in a global market where the effective management of information systems (IS) is an important contributory factor to the efficiency of organizations. In a similar way, Rosca et al. [27] argue that business rules which represent decisions about functional and non-functional requirements are requirements that arise from business objectives. In other words, business objectives determine the business rules which govern software systems.

\section{RE Decisions Framework}

The manageability of the requirements process is important as the quality of the process affects the quality of the product. By studying the decision-making process in RE activities in more detail, we can develop a better understanding of how the decision-making operates within the RE process. In our earlier work, we investigated the integration of classical decision-making models with RE process models [5]. Based on Anthony's [3] three level managerial decisions, we introduced and developed a framework that takes into account RE decisions in terms of product, project and business decisions [6, 7]. We pointed out that when investigating requirements decisions, the following components need to be considered: a) business process, objectives, and rules; b) stakeholders involvement; c) information requirements; d) RE decisions in three different organizational levels. We argued that business objectives determine also business processes and business rules. The following relationship is anticipated:

\section{Business Objectives $\rightarrow$ Business Processes $\rightarrow$ Business Rules $\rightarrow$ Software Product Requirements}

To describe three components in a decision context, we used a conceptual modeling technique that described and clarified the entities involved in the decision-making process in RE activities [7]. It should be emphasized that it was essential also to capture all relevant information during the RE process so that stakeholders can select the best alternatives when deciding on requirements. Hence, it was important to understand the relationship and interactions between business processes, stakeholders and RE decision types in terms of strategic, tactical and operational decisions and information usage.

In this article, we extend this work by discussing the framework for RE decisions in more detail and argue that consideration of RE decisions at three different management levels facilitates tighter integration between business objectives and RE decisions. This allows an appreciation of how the significance of aligning RE decisions requires an understanding of the relationships between business processes, the software product, the software project and stakeholders.

\subsection{RE Decisions in Three Perspectives}

Table 1 illustrates classifications of software requirements from three perspectives. In the first perspective, the classification is based on the major components of RE decisions, namely organizational-oriented (or businessoriented), product-oriented, and project-oriented decisions. The second perspective classifies the RE decisions into three management decisions, namely strategic, tactical (management control), and operational levels. In the third perspective, RE decisions are classified based on timeline in terms of pre-project decisions and in-project decisions. In Table 1, each cell includes examples of requirements activities and also provides some examples of the types of decisions that can be made at the different levels. 
Since the software applications need to support the business strategies, it is expected that ideally the following components are well aligned with each other.

- Perspective 1

- $\quad$ Business Decision $\leftarrow \rightarrow$ Product Decisions

- Product Decisions $\leftrightarrow \rightarrow$ Project Decisions

- $\quad$ Perspective 2

- $\quad$ Strategic Decisions $\leftarrow \rightarrow$ Tactical Decisions

- Tactical Decisions $\leftrightarrow \rightarrow$ Operational Decisions

- $\quad$ Perspective 3

- Pre-Project Decisions $\leftrightarrow \rightarrow$ In-Project Decisions

Although there may be a large group of stakeholders involved in a project, when it comes to the decisionmaking process, only a subset of the stakeholders participate e.g. product managers and project managers. Furthermore, a decision is rarely explicit. While the product requirements change during the development process, it becomes a challenge to meet project requirements if the development team has not participated in the decision-making process. The objective of the decision taxonomy in Table 1 is not to illustrate that decisions are isolated from each other, but to show the nature of these decisions in the context of software development. So far the research in RE has inadequately acknowledged the need to separate these three perspectives. To the best of our knowledge, this research is novel, in the sense that it clearly differentiates between the pre- and in-project levels in RE of software development and then establishes and builds on the connection between them.

Table 1. RE Decisions Framework (Adapted from [6])

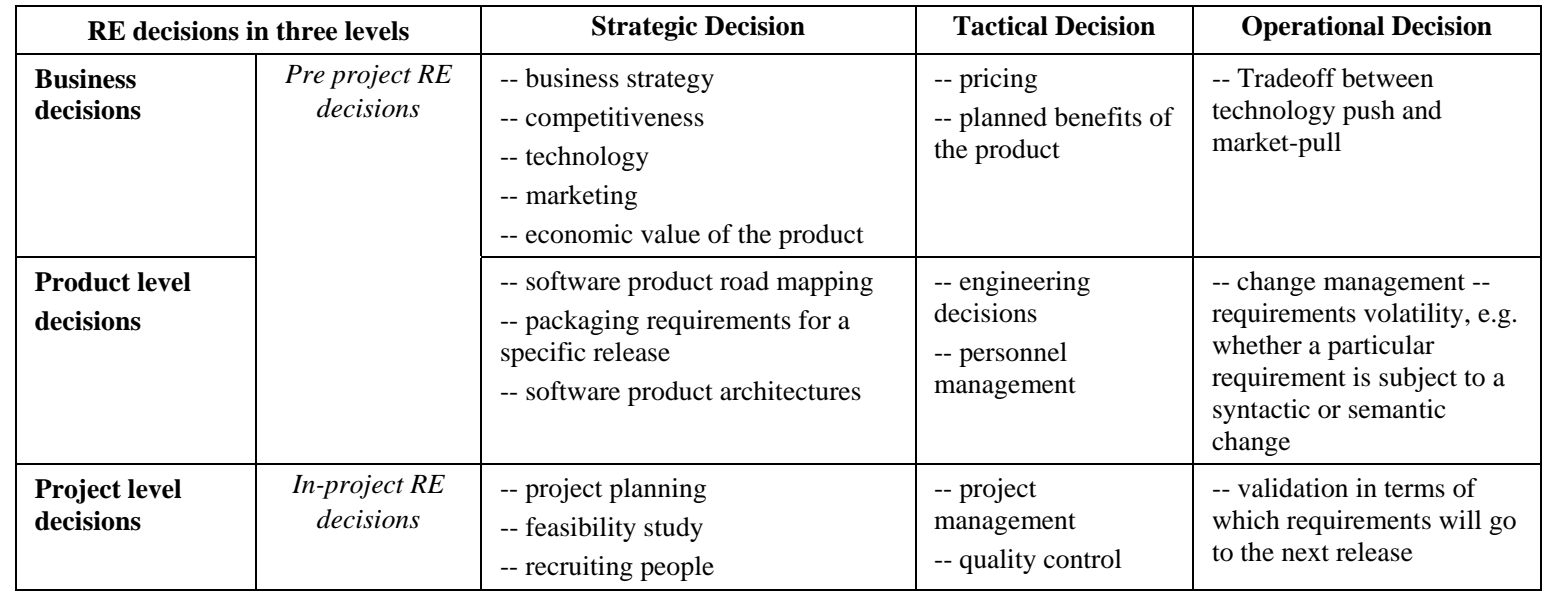

Note that we are currently conducting an empirical study related to the whole framework presented in Table 1. In this article, however, we focus on only project level decisions (in-project $R E$ decisions) and present empirical data from industry. Note also that the data were collected from a customer-specific project in which the customer was external to the development team.

\subsection{RE Project Level Decisions}

In many development models, it is assumed that RE is conducted up front. On the contrary, RE needs to be a continuous process throughout the project because requirements are volatile and any changes have to be handled within the project as they occur. Requirements at the product level must be packaged into parts that go into specific projects or releases of the software. It is important that requirements are ranked by priorities and selected based on their fulfilment of both product and organizational goals and strategies. Requirements may be chosen for implementation based on whether they fulfil the needs of a specific and important customer, or whether they potentially open up a new market segment to the organization [33, 34]. These requirements define 
the conditions under which the project will be run, including issues related to project planning, risk management, budget and cost.

- RE Strategic Decisions: Strategic decisions in requirements activities require creativity and opportunistic inputs, and should be based on an accurate understanding of current business processes and a detailed understanding of the software product. In the early stages of product development, developers need to decide which requirements to include in the next release and which ones to postpone to the following release. This decision is strategic from a product point of view, as some crucial features of the product, i.e. requirements, may signify the difference between gaining and loosing market shares.

- RE Tactical Decisions: Tactical decisions are about the implementation of strategic decisions. The decision maker is constrained by the availability of key resources, i.e. human and non-human resources. For example, a decision may involve answering the following questions: What sort of project management do we have? What are the planned benefits? What are the resource reductions? What are the product enhancements? What priorities are placed upon the achievement of these benefits?

- RE Operational Decisions: Decisions made during this stage involve a solution to a given situation. The activities involved here are concerned with the implementation of requirements. The information involved at this stage is quite detailed and arises from organizational sources. Decision makers may consider several tools and decision aids when making decisions. Operational control requires project managers to focus on delivery of the software product and meeting the project budget.

\section{Description of the Industrial Case Study}

The case study was a software development project undertaken at a large Australian insurance company. The company is one of the largest general insurers in the Australian market, with net profits in excess of 750 million AUD. The growth of the company has been in part facilitated via mergers and acquisitions of smaller insurance companies. One of the results of this expansion, from a strategic technology perspective, has been that the company now manages a number of disparate systems throughout the organization, each catering to the differing needs of the numerous general and specialized insurance products the company now provides.

The project commenced in January, 2005 and the data were gathered during the design phase of the project from January to July 2005. The project was classified by the organization as being large because its budget was in excess of 4 million AUD.

The development team undertaking the project was relatively young, having been formed as a result of a restructuring of the IT department approximately 18 months prior to the start of the project. In the team's first 18 months, it had maintained the stability of the insurance management system, having achieved a $99 \%$ uptime record prior to the start of the project. The team had managed also to implement several enhancements to the system during this period.

The objective of the project was to implement changes to both the claims and policy management modules of an insurance management system. The required changes were initiated by government legislation and hence, the majority of the requirements were non-negotiable.

The insurance company was required to implement the legislative requirements before a stated deadline in order to retain their license to operate. Three months after the project started the relevant government body had issued approximately 600 pages of official requirements documentation including 12 subsequent addendums to this documentation, bringing the total requirements to approximately 1,000 pages. As a result, requirements definition, design, and tracing were major activities in the project.

The relevant government department operated as an external stakeholder throughout the project via the issuing of requirements and addendums to these requirements. The government department did not at any stage become directly involved at the tactical or operational level in the project - the design, implementation and project management tasks were the sole responsibility of the development team at the insurance company. The government body was approached to discuss the decision-making activities from their viewpoint, but declined to be involved in the study. Therefore, this study is limited to explaining the decision-making process from the viewpoint of the development team at the insurance company. 
There were approximately 30 IT staff assigned to the project, in various capacities including Project Manager, Developers, Systems Analysts, Business Analysts, System Testers, User Acceptance Testers, and Business Sponsors. Approximately 50\% of the staff were full time IT professionals employed by the company and the remaining $50 \%$ were Senior IT Consultants contracted specifically for the project. The staff were hired or contracted on the basis that they possessed above 3 years experience in the insurance industry in an IT capacity.

The company undertaking the project maintained a uniform project management policy for all projects conducted by the IT department, including a standard development methodology. The project brief stated that the project would follow this methodology, which included the standard sequential software development life cycle phases, namely Requirements Definition, External Design, Internal Design, Coding, System Testing, User Acceptance Testing (UAT), and Implementation.

\section{Methodology}

In this paper, we use the framework illustrated in Table 1 to examine only the project-level RE decisions in the context of the case study described above. An investigation of SE and IS literature revealed that there is a large amount of IS research that investigates IS development decisions at the business level, e.g. literature that focuses on how to achieve competitive advantage with IS [24, 30,35]. There is also a large amount of research in SE that addresses product-level operational decisions, such as trade off analysis, [2, 23, 17, 28] and prioritization [18, 31, 32]. To the best of our knowledge, there is no existing study that specifically focuses on project level RE decisions.

The objective of this research was a) to identify project related key RE decisions at the strategic, tactical and operational levels, b) to examine to what extent RE decisions are aligned on these three levels. The analysis of RE decisions was facilitated by the case study. The relevant empirical data were gathered from analyze of existing documentation within the company and interviews with the project team. During the data collections, we focused on information, decision type, stakeholders and decision tools that were used in RE activities.

\subsection{Analysis of Existing Documentation}

The majority of the documentation was retrieved from a folder allocated to the project. Certain documents such as the project brief and meeting minutes had to be officially approved for release by the project manager. The documents were formatted and analyzed using the content analysis VBPro ${ }^{1}$ tool. The documentation utilized included:

- Project Brief: Standard statement of work required at the commencement of any project at the company to establish scope.

- Meeting Minutes: Minutes were kept from every meeting held in relation to the project and stored in the project folder on the shared drive.

- Official Memos: Most emails related to the project were sent to a standard email address that forwarded the message to all members of the project team

- Technical Manuals and Legislative Requirements: These consisted of over 1,000 pages of documentation which were available as a soft copy and thus were included in the analysis. Although the requirements were generic, certain sections did specifically refer to decisions the insurance companies reading the document had to make in order to satisfactorily implement the requirements. For example, "The Insurance Company must make a decision as to implement a unique policy identifier or a translation number for internal data integrity purposes."

- Business Requirements: These were standard project documentation produced and distributed by members of the project team.

- External and Internal Designs: These were standard project documents produced and distributed by members of the project team.

\footnotetext{
${ }^{1}$ VBPro is set of programs that analyses the text. http://mmmiller.com/vbpro/vbpro.html
} 
- Test Plans: These were standard project documents produced and distributed by members of the project team.

The content analysis procedure provided a description of the RE decisions identified from the documentation. Then, these decisions were thoroughly analyzed in terms of their impact and the stakeholders involved. In analyzing the decisions, we also considered which decisions would be the most appropriate for further investigation in the later stages of the research. For example, decisions concerning company sensitive information were excluded in line with request by the company involved. Decisions involving several stakeholders were given preference for later investigation, the rationale being several perspectives of the decision could be sought which may aid in collecting more detailed and richer data. Using this approach, we refined the data to arrive at a set of the key decisions made in the project.

\subsection{Interviews with the Project Team}

A set of interviews was carried out with the key project team members. The participants were selected based on their involvement with the RE decision-making process which included the project manager (PM), three systems analysts (SAs), two business analysts (BAs), and two developers and programmers (DVs).

Each interview lasted between 30-50 minutes, and all were carried out over a two-day period. The interviews were carried out in private meeting rooms at the company premises.

The interview script explored the characteristics of the decisions which had been identified in document analysis. The decisions which were the subject of each interview were tailored to the level of stakeholder involvement in the decision. For example, in the SA interview, the decisions discussed mainly focused around design issues raised in the project. The discussion covered 3-4 of the key RE decisions which had been identified in the document analysis. The discussion was semi-structured, that is, participants occasionally could continue discussions along the lines of their own volition, but were eventually brought back to the decision under consideration by the interviewer. The questions covered were tailored based on the participants' level of involvement in each decision. The questions sought to discover the role if any, that information, people and tools had played in the decision-making process in each of the examples raised. The participants were also asked some general questions about their individual perspectives on how strategic, tactical and operational decision-making activities were carried out in the project.

The interviews were recorded and later transcribed, apart from the PM interview, in which notes were taken by the interviewer. When the interview scripts had been transcribed, they were distributed to the interviewees who verified the transcript was an accurate account of the interview.

\section{Results and Discussion}

This section presents the raw data collected during this research study. Firstly, the data collected from the documentation analysis are presented, including the decisions that were identified from the documentation. Secondly, a discussion of specific instances of RE decision-making activities, which was collected from the focus group interview transcripts, is presented.

\subsection{Analysis of Existing Documentation}

The analysis of documentation allowed us to identify 5 main strategic, 10 tactical and 7 operational level decisions as illustrated in Table 2. There were also quite a number of decisions identified from the documentation that were inappropriate for discussion, due to their sensitive nature. In Table 2, in each of the decisions described, the name of each piece of functionality being considered has been replaced with a numbering system (Functionality A, Functionality B, etc.) in order to protect the anonymity and privacy of the company studied. In Table 2, the labels "S", "T" and "O" are assigned to strategic, tactical and operational decisions respectively. The last column of Table 2, "Discussed By" shows which decisions have been followed up through the interviews in this study. 
Table 2. Decisions discussed in the interviews

\begin{tabular}{|c|c|c|}
\hline Decision & Description & Discussed By \\
\hline S2 & Evaluation of the project's functional scope & - \\
\hline S3 & Alignment of the project management methodology with the organizations overall IT structure & - \\
\hline S4 & Decisions to improve the current system vs build/buy a new system & - \\
\hline $\mathrm{T} 2$ & Termination of testing team and reassignment of testing duties to systems analysts & PM \\
\hline T3 & Approval policy: individual email vs. workshop approach & PM \\
\hline T4 & Decision to include Functionality A in scope & SA \\
\hline T8 & Decisions on the choice of Solution for Functionality D & SA, BA \\
\hline T9 & Decision on choice of solution for Functionality F & BA \\
\hline $\mathrm{T} 10$ & Approach to recruitment of staff - decision to hire a majority of consultants for the project & - \\
\hline $\mathrm{O} 1$ & Day to day design decisions related to Functionality A & - \\
\hline $\mathrm{O} 2$ & Day to day design decisions related to Functionality B & - \\
\hline $\mathrm{O} 3$ & Day to day design decisions related to Functionality D & SA \\
\hline $\mathrm{O} 4$ & Day to day design decisions related to Functionality E & SA \\
\hline O5 & Interpretation of requirements into internal design, decisions on what to include or exclude. & SA, DV \\
\hline
\end{tabular}

The decisions identified in Table 2 offered some insight into the overall business objectives which the organization was aiming to fulfill as a result of the project. We identified most of the strategic decisions from the project brief and the tactical decisions from the correspondence between the project team, such as the meeting minutes or official memos. The majority of the tactical decisions were related to selecting the functionality to include in the final solution, and these decisions were largely driven by schedule and cost pressures. Significantly, the strategic decisions did resonate quite as strongly where tactical decisions were discussed in the documentation. For example, decisions to include pieces of functionality in scope were largely accompanied in the documentation by a justification such as "it was in the functional scope according to the government requirements", of which the specific functionality was related to a strategic objective.

The operational decisions identified from the documentation were, by and large, related to decisions stakeholders had to make regarding the design of certain pieces of functionality. The government requirements documents were the major source of the operational decisions because in most cases the requirements were specified at a low level to ensure compliance with the legislation. To a large extent the government documentation implied the decisions that needed to be made. The fact that the majority of the operational decisions were identified as being design decisions was not unexpected. The requirements were static, and implementation of the requirements into the existing insurance management system required a degree of interpretation, and hence, operational decision-making.

\subsection{Interviews with the Project Team}

The interviews aimed to develop a deeper understanding in the social context of the decisions that identified in the document analysis. From the decisions identified in the document analysis, decisions were discussed with the project team. Each interview covered 3-4 key decisions from each organizational level, according to the time available. 
For example, S5 involved the PM utilizing information, in the form of government requirements, to make a decision about quality benchmarks to be put in place for the project. The PM was recruited to the project at its commencement and was assigned exclusively to manage the project studied. He was the decision maker in a number of the strategic and tactical decisions.

T2 involved the termination of the testing team. In making this decision, the PM considered the information he had relating to the project deadlines, and evaluated this information in conjunction with the notification he received from the government stakeholder regarding an extended deadline. T3 involved the PM making a decision based on the opinion of a business manager and an SA, both of whom undertook similar amounts of interaction with the decision maker. However, the interviews suggested that the business manager was more influential in the decision-making process than the SA. T8 involved the choice of a solution for a particular piece of functionality required by the government legislative requirements. To illustrate the complexity of this decision, it is presented from the perspective of both a systems analyst and a business analyst.

O3 was concerned with making day-to-day design decisions in the process of completing the design documentation in relation to functionality D. O7 was concerned with making day-to-day design decisions for the business in relation to functionality $\mathrm{F}$ which involved modifying existing functionality.

This paper presents only a subset of the decisions that were made. These decisions were selected based on the amount of data collected on the decisions discussion in the interviews and the relative significance of the decisions in terms of their interaction with decision-making processes at different managerial levels of decisionmaking. For example, S5 was selected for discussion because several tactical decisions were made, according to the stakeholders, based on the outcome of this higher-level strategic decision. Decisions are also studied in terms of intelligence, design, and choice phases [29].

S5 - Quality benchmark requirements for the final solution: Strategic decisions were difficult to discuss with the project stakeholders because few stakeholders had been involved in them and most of these decisions were of a nature that was sensitive to the company thus were not appropriate for discussion in this paper. However, the PM did discuss the strategic decision of quality benchmarks for the final solution. The PM needed to establish quality benchmarks for the project to ensure the project remained in scope and was delivered on time. While the PM felt both information and people were influential in this decision, he believed that information had a relatively stronger influence. The PM indicated that he had specified project parameters from upper management and the government in terms of cost and schedule. Accordingly, his strategic objectives were affected by these parameters:

You could say the [government] requirements were probably the biggest factor [in the decisionmaking process] because they set out exactly what we had to do very clearly so we had to work the schedule around them and keep costs acceptable to the business.

The PM explained that he had specified project parameters from upper management and the government in terms of cost and schedule, so his strategic objectives were affected by those parameters. An interesting observation of this decision is the grounding of the decision in information, in this case the government requirements, because "they set out exactly what we had to do very clearly so we had to work the schedule around them." Essentially, the PM went onto describe how he felt the best approach was to achieve the basic government requirements word-for-word, and how to add extra functionality if the schedule permitted. This approach of grounding the decision in a specific rigid attribute was consistent across the strategic decisions made in the project. This approach makes sense from the point of view of ensuring stability in the requirements. Furthermore, this approach demonstrates "ensuring requirements are aligned with business objectives" as described in [7]. A final interesting observation of the use of information to ground the strategic decision was that it makes the rationale of the decision explicit in the context of which it was made, and the information serves as a record of this rationale. Recording the reason for a decision at the time it was made could serve several future purposes should attributes of the project change at a later date. 
T8 - Decision on choice of solution of Functionality D: To illustrate the complexity of this decision, it is presented from the perspective of both an SA and a BA. From the perspective of BA1, this tactical decision was concerned with

deciding on which solution for [functionality D] would satisfy the business people I represent and would be feasible for IT to build in the project timeline." SA1 saw this tactical decision as consisting of "choosing a solution for the [functionality D] process to meet the government's new requirements and we knew any of the choices was going to have a major impact on the end users [functionality D] processes.

The SA viewed the intelligence and design phases of the decision being dominated by the PM, who was in turn heavily influenced by a small amount of information from the government requirements, and less influenced by a larger amount of interaction with the SA. The PM in turn influenced the business manager, who was responsible for the choice phase of the decision. According to the SA, the business manager was strongly influenced by a large amount of subjective information which flowed from other external business stakeholders, and influenced to a lesser extent by direct meetings with the other business stakeholders themselves. The SA also suggested they utilized decision tools to a limited extent in this instance. On the other hand, the BA's view of this decision differed considerably from that of the SA. The BA saw themselves utilizing both the SA and the government requirements to influence the BA at the 'choice' phase of the decision. Presumably this was carried out with the goal of influencing the outcome of the 'intelligence' and 'design' phases of the decision which had been dominated by 'other business stakeholders' or as they were referred to by the BA, "users who just were serial decision hijackers.”.

07 - Design decisions related to Functionality F: To illustrate the complexity of this decision, it is presented from the perspective of both an SA and the DV. The SA saw O7 as quite complex. In his interpretation, the SA and the DV utilized information from the government stakeholder and were influenced by other business users, as well as each other. The other business users influenced the intelligence and design stages of the decisionmaking process in conjunction with the systems analyst. Similarly, the DV influenced the choice stage of the operational decision in conjunction with the SA. Interestingly, the DV's view of the decision was more simplistic. He saw both the DV and the SA having influenced all three stages of the decision-making process in conjunction with each other but free of further influence from other stakeholders. The developer also utilized decision tools to some extent, and both stakeholders heavily utilized information from the government stakeholder in making the decision.

\subsection{Relationships between the Decisions}

Using the interview data, we established the relationships between decisions at different levels of the management decision-making framework, as illustrated in Figure 1. The legend for this figure is presented in Appendix A. This figure has been included to illustrate the alignment between strategic, tactical and operational RE decisions in terms of how decisions at different levels of the management can affect one or more of the decisions at different levels.

Many of the decisions were related by the fact they dealt with similar tasks in the project at different managerial levels. For example, FD was dealt with at an operational level by the design decisions represented by O3. The choice of solution for FD was dealt with in T8. Finally, S5 is related to quality benchmarks that affected both the solution chosen at the tactical level and the design decisions made at the operational level. These three decisions at the different management levels, which dealt with similar requirements in the project, are referred to as a decision 'set'. In Figure 1, two decision 'sets' (left and right hand side of Figure 1) are presented to illustrate the complexity of RE decision-making in this project and to understand how different levels of management decision-making affect each other. The purpose of the decision set is to illustrate the similarities and differences between the types of activities that occur at the different levels of management decision-making. Each decision is examined in terms of intelligence, design and choice [29]. 


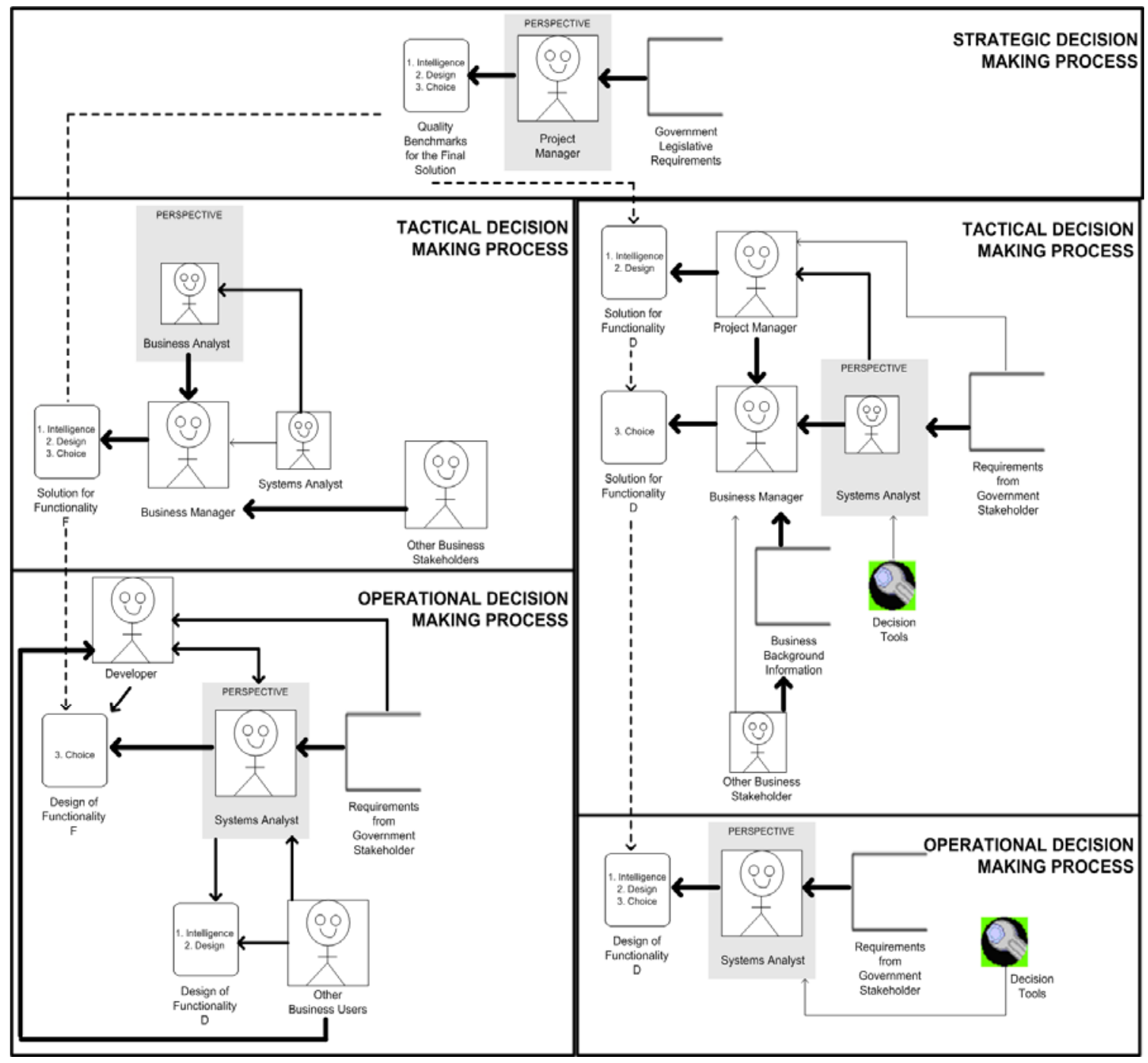

Figure 1: Representation of decisions related to functionality D and F

The left hand of side Figure 1 shows decision set 1, which is related to FD in which the perspective of the SA was chosen to represent the tactical and operational decisions. SAs were selected because these stakeholders had provided their perspective on the decision-making activities related to this piece of functionality at more than one managerial decision-making level. We believe this contributes to the cohesiveness of the data used in the model and avoids the complexity of attempting to explain the differing interpretations between stakeholders. Note that the dashed line illustrates the continuation of a decision at three different management levels.

The right hand side of Figure 1 shows decision set 2 which is related to functionality $F$ in which the perspective of the BAs were chosen to represent the tactical decisions and the systems analyst's perspective was chosen to represent the operational decisions. These perspectives were chosen because both perspectives acknowledged the existence of the other business stakeholders and/or users as an influencing factor in the decision. The DV's perspective did not acknowledge the 'other business users/stakeholders'.

\subsection{Discussion}

The modeling of the key RE decisions illustrated the factors that influenced the project decisions. The following key observations are made from this study. 
The findings showed that the perspective of each project team members affected his/her interpretation of the decisions making process. While this observation might seem fundamental, it has implications for stakeholders being supplied with relevant information at the right time in the decision-making process. For example, in $\mathrm{O} 7$ based on the interpretation of the DV's perspective on decision, they were not aware of the existence of the other business users in the RE decision-making process. It is possible that if the DVs knew of the additional stakeholders involved in the project, they could have offered support to these stakeholders. Unfortunately, in the project studied, many of the decisions suggest a degree of spontaneity in the RE decisionmaking process, many of the stakeholders were not adequately briefed on the scope of the decision or the relevant parties involved and so were less able to offer relevant decision support where it was required. This observation underscores the need for providing stakeholders with the relevant support required based not only on their role, but also on the type of decision they are engaged in i.e. in strategic, tactical and operational level.

The level of interaction between team members, and a stakeholder, information, or decision tool is not necessarily proportional to the degree of influence that the attribute eventually places on the decisionmaking process. For example in T9, the BAs believed that they had a very high level of interaction with the decision maker. However, the interview results showed that they did not have as high as influence on the decision-making process as the "other business stakeholders". This observation highlights the need to engage the right stakeholders in the RE decision-making process according to the type of decision and its possible implications. Engaging the wrong stakeholders in the decision-making process appears in this project to have added unnecessary complexity and misunderstanding between the stakeholders.

The entire project team's view of the RE decision-making process did not always align with the specific instantiations of the decisions articulated by the stakeholder in each interview. The examination of the company's existing documents and interviews with key software team members revealed substantial truths about what actually worked and what didn't in this particular project. Although the concept of supporting internal information to improve operational decisions and to achieve business objectives was well recognized by the project team members, the alignment of in-project decisions with pre-project decisions was perceived as complex and challenging. Not surprisingly, in-project decisions were inextricably intertwined with product and business decisions. Most importantly, the main challenge for the software team members was to consider aligning their decisions not only with their own business objectives but also with the customer's business objectives. During the interviews, we observed that not all the perceptions of the project team aligned with specific instances of each level of decision-making. There was better alignment between SAs and DVs than between SAs and BAs. Furthermore, BAs found it difficult to align the requirements with the business objectives when the customer was external when the communication is a major problem. In other words, alignment of strategic decisions with tactical decisions was more challenging than alignment of tactical decisions with operational level decisions. This observation conveys the need to ensure that RE decisions are aligned with business objectives, decision makers are provided a decision criteria, and relevant key information for unstructured problems. If stakeholders feel unsure of the basis for a RE decision, they should feel comfortable in questioning how the decision aligns with the business objectives. An unwillingness or inability to justify a RE decision based on the business objectives may highlight an area of the project which needs further analysis by management stakeholders.

Note that this observation is somewhat limited as the project we studied was large, complex, and customerspecific in which access to the customer was not possible. Thus, comparison of decisions in different levels was restricted to perspectives of the development team. However, the findings indicate the complexity of decisions in-project as well as the need to align the in-project decisions with decisions on other levels as expressed by the framework presented in Table 1. The complexity of the decision-making process related to the different levels and the different types of decisions shows the need for a framework to enable mapping of decisions on different levels and different types to each other.

\section{Conclusion}


The growth in strategic importance of IT brings with it the need for tools, techniques and processes to be integrated with software system requirements so that they are aligned with the strategic business objectives and business models of the organizations they support. Business change is a part of system development. As systems become more integrated and involve more users from diverse backgrounds, software developers are pressured to understand the implications of their decisions in relation to cost/benefit analysis, particularly during early life cycle activities.

This research focused on RE activities, provided a framework that addresses the decision-making process within early life cycle activities and investigated RE project level decisions in an industrial case study context. This research also examined to what extent practitioners aligned their decisions with business objectives in this particular case study. The key RE in-project decisions were identified in terms of the three management levels as strategic, tactical and operational decisions. Furthermore, the RE decision-making activities were modeled to provide further illustration of to what extent and how RE decisions were carried out in the project.

Our findings showed that project level decisions were heavily influenced by business and product requirements. We identified several shortages in RE project decisions through the interviews that were conducted with PM, SAs, BAs and the DVs. Not surprisingly, many of the decisions had an ad hoc nature, and the decision-making process and the decision criteria were not well defined. Furthermore, in some cases, the stakeholders were uninformed about the decision, decision process and the outcome of the decision. Decision leader and decision executors were not necessarily the same people. The findings showed that stakeholders had a major influence in-project decisions. However, in some cases, due to time pressure and ambiguity in the process and the decisions, the project decisions were dominated by some business stakeholders or, as they were referred by one of the BAs, decision hijackers. It was difficult to align the requirements with business objectives when the customer was external. In other words, not all the perceptions of the project team aligned with specific instances of each level of decision-making. A lack of understanding of the business strategy and lack of communication between business level, product level and project level stakeholders were among the problems that were highlighted in this study.

In order to support RE activities during software development, it is important to present some solutions to the problems mentioned above. While it is not necessary to control or record all the decisions that were performed during the RE activities, we believe that the following issues need to be handled in software development:

- Provide support on different perspectives of decisions including business, product, and project level to stakeholders and/or decision makers

- Provide decision criteria for the above perspectives e.g. market timing, customer value, company preference. Although many decisions are subjective and intuitive, the criteria will help the decision maker to formulate their decision

- Provide support to improve reliability of decision-making process and/or the outcome of the decision. The decision-making process and the uncertainty handling process need to be well defined and the two processes need to be linked to a decision criteria

- Provide to decision makers an environment in which they can share information and knowledge so that they can have a mutual understanding and perspective about the software product.

An understanding of RE as a decision-making process seeks to help organizations ensure requirements decisions are aligned with business objectives and provide enhanced decision support for RE activities. The contribution of this paper lies in the development of RE decision-making framework and detailed analysis of the alignment of in-project decisions. We expect that this framework will enhance the body of current academic knowledge on the subject of RE decisions. The research can also benefit practitioners in this field, by providing an understanding of the factors that influence effective decision support to RE in-project decisions. Similarly, software developers can better plan for their RE activities. The development of an RE decision-making framework can be used to facilitate an understanding of the RE process. In practical terms, this means future researchers can utilize this study as a guide for researching the RE decision-making processes inherent in other software development projects. It is also envisaged that the company involved in this study will be able to use this research as a benchmark for future investigations into RE decision-making processes. Specifically, light will be shed on (a) which stakeholders engage in the decision-making process and the types of decisions they make; 
(b) the complexity of certain decisions with respect to specific areas of functionality i.e. functionality A, B, C etc.; (c) the process followed in making each type of decision i.e. strategic, tactical and operational and how to improve these processes with the aid of additional information, stakeholders and decision tools.

\section{Reference}

1. B. Alenljung and A. Persson, Supporting Requirement-based Decision-making in the Software Engineering Process: A Position Paper. REFSQ’04 (Riga, Latvia, 2004), pp.63-68

2. I. Alexander, Initial Industrial Exploration of Misuse Cases in Trade-Off Analysis Proceedings of the IEEE Joint Conference on Requirements Engineering, (2002), pp.61-70

3. R.N. Anthony, Planning and Control Systems: A Framework for Analysis, (Harvard U., Boston, USA, 1965)

4. A. Aurum and E. Martin, Managing both Individual and Collective Participation in Software Requirements Elicitation Process, (14th Int. Symp. on Computer and Information Sciences, Kusadasi, Turkey, 1999), pp.124-131

5. A. Aurum and C. Wohlin, The Fundamental Nature of Requirements Engineering Activities as Decision-making Process. Journal on Information and Software Technology, (Elsevier Science, 2003), 45(14): 945-954

6. A. Aurum and C. Wohlin, Requirements Engineering: Setting the Context, in Engineering and Managing Software Requirements, eds. A. Aurum, C. Wohlin (Springer-Verlag, Germany, 2005) ISBN: 3-540-25043-3

7. A. Aurum and C. Wohlin, Aligning Requirements with Business Objectives: A Framework for Requirements Engineering Decisions. Workshop on Requirements Engineering Decision Support, REDEC'05, (Paris, France 29 Aug2nd Sept 2005)

8. B. Boehm, Value-Based Software Engineering. ACM SIGSOFT, Software Eng. Notes, (March, 2003) 28(2): 1-12

9. BSC'04, The challenges of complex IT projects. Royal Academy of Engineering and the British Computer Society, ISBN 1-903496-15-2. Access on 20th October 2004 http://www.bcs.org/BCS/News/PositionsAndResponses/ Positions/complexity.htm

10. R.E.M. Champion and T.T. Moores, Exploiting an Enterprise Model during Systems' Requirements Capture and Analysis, International Conference on Requirements Engineering, (1996), pp. 208

11. T.H., Davenport, Process Innovation, Harvard Business School Press, (Boston, MA, 1993)

12. M. Davson, Are Software Processes Business Processes Too? Panel Session of the Proceedings of the 3rd Int. Conference on the Software Process, (Reston, Virginia, USA,1994), pp. 177-178

13. R., Evans, S. Park and H. Alberts, Decisions not Requirements: Decision-Centered Engineering of Computer-Based Systems, Workshop on Engineering of Computer-Based Systems (Monterey, CA, USA, 1997), pp. 435-442

14. S.R. Faulk, R.R. Harmon and D.M. Raffo, Value-base Software Engineering: A Value-driven Approach to Product-line Engineering, 1st Int. Conf on Software Product-Line Engineering, (Denver, Colorado, August, 2000)

15. C.W. Holsapple and A.B. Whinston, Decision Support Systems: A Knowledge-Based Approach, (West Pub. Comp, USA, 1996)

16. L. Jiang and A. Eberlein, Decision Support for Requirements Engineering Process Development. Canadian Conference on Electrical and Computer Engineering, (2003) pp. 1359-1362

17. H. Kaiya, K. Sasaki, Y. Maebashi, and J. Kaijiri, Trade-Off Analysis between Security Policies for Java Mobile Codes and Requirements for Java Application, Proceedings of the $11^{\text {th }}$ IEEE International Requirements Engineering Conference, (Monterey Bay, CA, USA, 2003)

18. J. Karlsson and K. Ryan, Prioritizing Requirements using a Cost-Value Approach, IEEE Software, (1997), 14: 67-74.

19. M. Lubars, C. Potts and C. Richter, A Review of the State of the Practice in Requirements Modelling. IEEE RE Conf, (San Diego, USA, 1993)

20. S. Maurice, G. Ruhe, O. Saliu, A. Ngo-The and R. Brassard, Decision Support for Value-Based Software Release Planning, in Value Based Software Engineering, eds. S. Biffl, A. Aurum, H. Erdogmus, B. Boehm, P. Grunbacher (Springer, Germany, 2005) ISBN 3-540-25993-7

21. L. Neumann-Alkier, Think Globally, Act Locally - Does it Follow the Rule in Multinational Corporations? 5th European Conf. on Information Systems (Cork Publishing Ltd, Cork., 1997), pp. 541-552

22. A. Ngo-The and G. Ruhe, Decision Support in Requirements Engineering. in Engineering and Managing Software Requirements, eds. A. Aurum, C. Wohlin (Springer-Verlag, Germany, 2005)

23. T. Pasternak, Using Trade-Off Analysis to Uncover Links between Function and Non-Functional Requirements in UseCase Analysis, Proceedings of the IEEE International Conference on Software-Science, Technology \& Engineering (IEEE Computer Society Press, 2003)

24. P. Pramongkit, Strategic IT Framework for Modern Enterprise By Using Information Technology Capabilities, IEEE Proceedings of International Engineering Management Conference, (Cambridge, United Kingdom, 2002)

25. R. Regnell, B. Paech, A. Aurum, C. Wohlin, A. Dutoit and J. Natt och Dag, Requirements Mean Decisions! - Research Issues for Understanding and Supporting Decision-Making in Requirements Engineering, 1st Swedish Conference on SE Research and Practice, (Ronneby, Sweden, 2001), pp. 49-52 
26. C. Rolland, C. Souveyet and M. Moreno, An Approach for Defining Ways-Of-Working, Information Systems, ( 1994) 20(4): 337-359

27. D. Rosca, S. Greenspan, M. Feblowitz and C. Wild, A Decision-making Methodology in Support of the Business Rules Lifecycle. IEEE RE Conf, (Annapolis, MD, USA, 1997), pp. 236-246

28. G. Ruhe, A. Eberlein and D. Pfahl, Trade-off Analysis for Requirements Selection. Journal on Software Engineering and Knowledge Engineering, (2003), 13: 345-366

29. H.A. Simon The New Science of Management Decisions. (Prentice Hall, Inc. Englewood Cliffs, NJ, USA, 1960)

30. A. Song, and H. Li-Hua, Comparative Study of Obtaining Competitive Advantage from Information Technology, IEEE Proceedings on International Conference on Services Systems and Services Management,. (13-15 June 2005), 1:19-23

31. J. Yen, J. and F. Liu, A Formal Approach to The Analysis of Priorities of Imprecise Conflicting Requirements, IEEE Proceedings of 7th International Conference on Tools with Artificial Intelligence, (5-8 Nov. 1995), pp. 164 - 167

32. K.E. Wiegers, First Thing First: Prioritizing Requirements, Software Development, (September 1999)

33. C. Wohlin and A. Aurum, Bundling Software Requirements to Create Product Value. in Value-based Software Engineering, eds. S. Biffl, A. Aurum, B. Boehm, H. Erdogmus, P. Grünbacher (Springer, Germany, 2005)

34. C Wohlin and A. Aurum, What is Important when Deciding to Include a Software Requirement in a Project or a Release? 4th International Symposium on Empirical Software Engineering, (Noosa Heads, Australia, 17-18 Nov 2005) pp. 246-255

35. J. Zurcher, Evaluating the Contribution of Information Systems to Organisational Competitive Advantage, IEEE International Conference on Systems, Man, and Cybernetics, (11-14 Oct. 1998) 3: 2523 - 2528 


\section{Appendix A.}

We used a hybrid model of the traditional data flow diagram (DFD) to represent a selection of the RE decisionmaking activities in the project. A DFD essentially consists of four symbols, namely: an entity (square), a data flow (line), a process (rounded square) and a data store (open rectangle) as illustrated in Table 3. Since the traditional format of a DFD does not clearly communicate influence of different factors on each other, we added two new elements to the notation to form a hybrid data flow diagram suitable for representing the RE decisionmaking activities in the project. Specifically, we included the following additional rules in the notation:

Table 3. Hybrid DFD notation used to represent RE decision-making activities

\begin{tabular}{|l|l|l|}
\hline Symbol & Meaning & Representation \\
\hline & Human Figure & $\begin{array}{l}\text { Stakeholders who were involved in } \\
\text { the decision-making process. }\end{array}$ \\
\hline
\end{tabular}

Table 4. Representation of the amount of interaction between two entities

\begin{tabular}{|c|l|}
\hline $\begin{array}{l}\text { Size of data flow line } \\
\text { in diagram }\end{array}$ & $\begin{array}{l}\text { Level of } \\
\text { interaction } \\
\text { between entities }\end{array}$ \\
\hline Low \\
\hline
\end{tabular}

- The size of the data flow line represents the volume of interaction between the two entities. As there is no scientific measure of the volume of interaction, the thickness of the line is a representative measure only based on the perception of the researcher (Table 4)

- The size of an entity represents the influence the entity was perceived to have on the decision-making process.

- The degree of influence was assigned a level of low, medium or high (based on quantitative data that was collected). This value influenced the size of each of the entities in the diagram

- For the data captured by the focus group interviews, the degree of influence of each decision attribute was assigned a level of low, medium or high according to the analysis of the interview data.

- For the purposes of clarity, we substituted the "entity" square for a human figure - in order to represent people, or a spanner - in order to represent tools, as appropriate. This resulted in a set of entities and their representations. 